\title{
The Role Embodied Cognition Plays in Situation Model
}

\author{
Jingting Wang ${ }^{1,}$ Zitong $\mathrm{Li}^{2}$, Zilin Qiao ${ }^{3, *}$ \\ 1'International trade and economy, Ningbotec University, Ningbo China, Elena_number9@163.com \\ ${ }^{2}$ English, Beihang University, Beijing China, lizitong001003@163.com \\ ${ }^{3}$ Georgetown Preparatory School, Washington D.C. America, Ziling0523@outlook.com
}

\begin{abstract}
At the beginning of our discussion, this paper depicted and reviewed the concept of modality-specific simulation and introduced the situation model. We further discussed the relationship between these two concepts and designed an experiment aimed to reach out the evidence that can be used to testify the function of situation model in the modalityspecific simulation. But we didn't actually operate the experiment. The entire design is constituted by two separate parts. For the first experiment, we prepared two kinds of articles that only one of them making sense (all articles have the same verb), and monitor the modality-specific areas by MRI. A time keeper is also involved in this experiment. We predict that activation will be stronger and the time will cost less for the reasonable ones. As soon as this prediction being verified, showing that a connection exists between the forming of situation model and embodied cognition, the second experiment will be presented. All of the sentences presented in experiment two can make sense but with different nouns, which means that the comprehenders are facing various situations in a reasonable way. We use TMS in this experiment and predict that modality-specific areas will be activated. If embodied cognition plays a role in the formation of one situation, it should be accessible for the others. Those two experiments mentioned above are still in the design-stage and not being performed. We hoped that our design can inspire you for some ideas within the area of situation model.
\end{abstract}

Keywords: component, situation model, embodied cognition, experiment.

\section{INTRODUCTION}

The discussion toward how our brains deal with the input and output of language was started from last century, when the concept of disembodied cognition was first suggested. Scientists described the process of language-understanding for human-beings as the program played for computers, giving out output corresponding to the input without the necessity to understand the meaning of the words. [16] The claim was questioned by some scientists who hold the idea of embodied cognition.Unlike the previous view, the embodied cognition suggested that when people operating stimuli from outside, conceptual representations were grounded in modality-specific areas through ways like re-enacting the states. [13] And some evidence from the fMRI images has proved the existence of sensory-motor actions.[12] Barsalou informed the perceptual symbol system which suggested that language,memory and thought are grounded in sensory-motor system. The perceptual symbols are reenactment of their indications and able to form simulators which play the role of specifying the concepts in the texts.[11] In the field of embodied cognition, Barsalou's perceptual symbol system is still very popular in recent years.

Situation model is a mental representation of the conditions or situations of an event. We function out the situation in our brains while not physically being there.[16] Segal described the process of how people create the situation model when reading texts (Segal, 1995, p.65), and Zwaan generalized the perspective of "deictic center"(e.g. Duchan, Brunder, \& Hewitt, 1995) in 3 dimensions, in temporal, spatial and psychological. Recently, LASS theory is also popular and widely testified. 


\section{DISCUSSION OF RELATIONSHIPS BETWEEN SITUATION MODEL AND MODALITY SPECIFIC SIMULATION}

\subsection{Further discussion of situation model}

A large number of embodied theories of cognition hold that the actual mechanisms underlying language comprehension is hypothesized to perform mental simulations of its content, the situation model.

Creating a situation model is rather constructing the situation than merely reappearing the literal text. Through this process, people tend to reconstruct a reasonable situation that is correspondent to the given texts. Loftus and Palmer[14] successfully overruled some subjects's perception by changing verbs in the sentence of the test, which shows how easy it is to manipulate one's memory by using situation model. We were deeply impressed by the idea that the memory can be overruled simply by changing single word in the text. First, it implies the idea that people were more confident with the presented texts rather than their memory. Then, people prefer to imaging a suitable situation for the text than checking out their minds. Although the experiment was unable to completely verify the existence of situation model, it did inspire us a lot. For example, what it explained in the paper revealed our thinking habit and dependent on short-term memory and longterm memory. We have a tendency to believe what is given by an authority. This implication may be an acceptable explanation for some social phenomenon and that we are easy to be manipulated.

Situation models will make the text-related scenery as real as possible. There are increasing evidence for this idea. In spatial consideration, when identifying the object that was described to be relatively far from the protagonist in the text, readers have a longer reaction time completing it than identifying the object that was described to be closer to the protagonist [4]. Another research found that when readers get more familiar with the space structure described in the text, it will be easier for them to form situation models of the stuff in this space structure [5].

Scientists also found that when we're understanding texts, our comprehension are likely to be influenced by the content. People's understanding towards 'approach' is different in 'The tractor is just approaching the fence' and in 'The mouse is just approaching the fence' [7], and the goal that the protagonist had not done was easier to be detected than the goals that had just been done [9]. it was also found that people are quick to infer the behavior of protagonists in a causal way [3]. People have their mental representation of the state of affairs according to the situation model. When the texts violate the stereotypes in our daily life, they need more effort to understand them. [1]
Compared to individual experience's role in enhancing situation models, coherent input conditions can also help us form coherent situation models. Researches showed that words' denotation can help people with the formation of situation models, [7] and with the inference for the actions of the protagonist. [8]

Moreover, when understanding action words, relative motor systems will be activated and we don't need to do the action at all. [12] For example, handrelated motor areas will be activated when we are understanding hand-related action verbs, and when understanding leg-related action verbs, leg-related motor areas will be activated. Although researches on situation models' properties and functions are numerous, there was still limited evidence about whether the process of operating situation models involves modality-specific areas, or situation models are implemented in modalityspecific cognition.

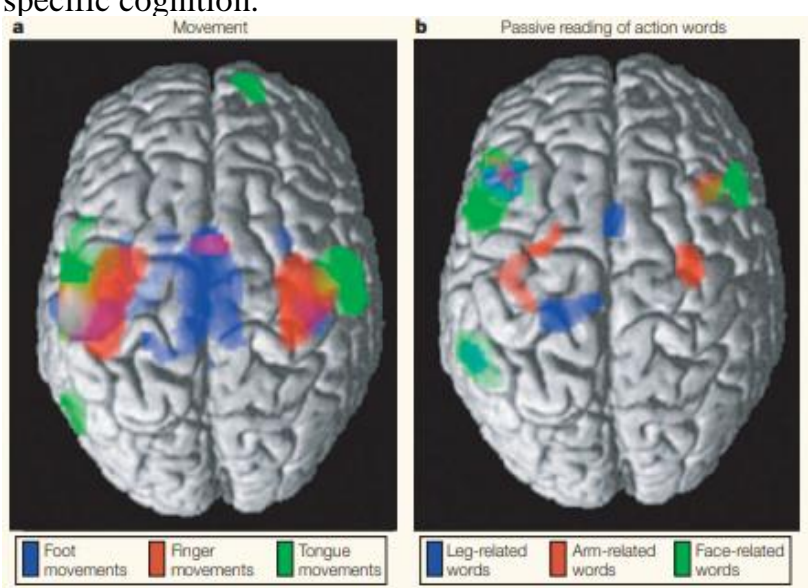

Figure 1 Brain mechanisms linking language and action by Friedemann Pulvermuller [12]

\subsection{Discussion of relationships between situation model and modality specific simulation}

Existing researches are unable to prove that situation models are embodied. Researchers found no overlapping areas between the early processing of specific properties and later formation of situation models, and only found a significant bound between the early word association and late situation generation [13]. This experiment failed because their hypothesis and results didn't match well and the reaction time was so long that the result is unacceptable. Moreover, they proposed that simulation and language procession were two separate processes, but in fact, it remained unclear.

Our design is based on some findings by previous researches. The comprehension of action words that are semantically related to different parts of the body may activate the motor system in a semantic manner [12], so we will use MRI to test out the activation of the textrelated modality-specific areas. (E.g., to test the armrelated area when the participants are presented for "the 
teacher grabbed a cake on the desk"; to test the leg area when participants are presented with the sentence "The teacher kicked the ball on the floor"). We need to focus on whether modality-specific areas will be more activated and our subjects will have faster reaction time when forming situation models in text understanding or not. Therefore, the hypothesis one for the whole experiment is that the activation will be stronger and time will spend less when forming situation models in text understanding. Once the prediction being testified, it can be concluded that the embodied cognition plays a role in forming situation models. Moreover, the hypothesis two holds the idea that the activation level and reaction time are the same no matter what the scenery is. It can be concluded that the A-model (computer model) theory is correct.

\section{EXPERIMENT}

\subsection{Experiment 1: whether modality-specific areas are involved in the forming of situation model}

\subsubsection{Participants:}

- native English speakers (even amount of female and male)

\subsubsection{Materials:}

- There are 48 sets of action descriptions.

- Half of the items describe the situations that correspond to the situation model (E.g.The teacher grabbed some cakes on the desk). Half of them do not (E.g.The teacher grabbed some words on the blackboard).

- The items are semantically related to different parts of the body that described hand action (e.g. to grab) and facial action (e.g. to eat). It is noted that the quantities for each kind of words is even. All of them used concrete expressions.

- Time keeper and MRI

\subsubsection{Procedure:}

All 48 descriptions are presented to participants one at a time, participants are asked to verify the grammar correctness of each sentence. The word "ready" appears in the center of the computer screen for two seconds immediately before each line is shown. The participants make their decision by clicking the button on the screen. If they think the sentence is correct in grammar, they will click the "yes" icon; If they think the sentence is incorrect in grammar, they will click the "no" icon.

To estimate the reaction time, the recording of time will begin as soon as the "ready" disappears. It will continue until the participants make their decision. At the same time, we use MRI to monitor the activation in the modality-specific areas. Both the reaction time and the extension of the activation are concerned in the experiment.

\subsubsection{Prediction:}

Based on the Experiment one, we have two predictions.

- Modality-specific areas will be more activated and have faster reaction time when subjects are presented with reasonable texts.

- The activation level and reaction time of modality-specific areas will be the same no matter what the scenery is.

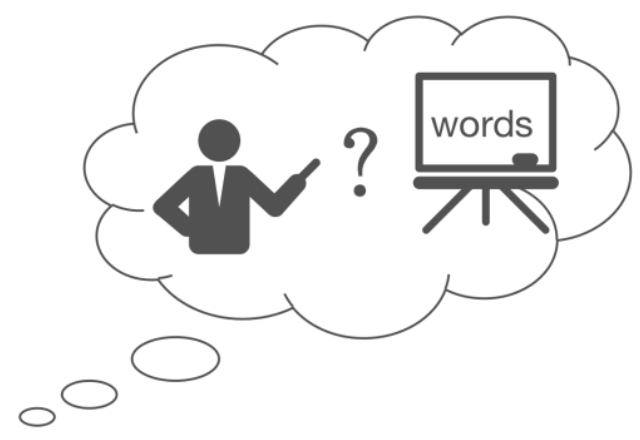

Figure 2 The unreasonable situation designed for experiment one

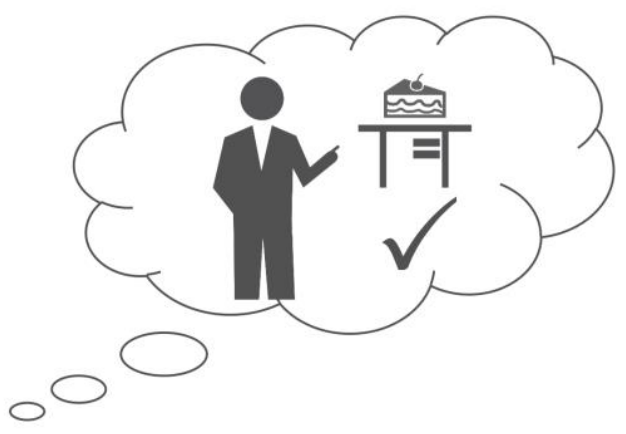

Figure 3 The reasonable situation designed for experiment one

\subsection{Experiment 2: What roles do modality- specific areas play in the forming of situation models?}

Experiment two is based on the experiment one. Once the result of experiment one finds out that the activation is stronger under the reasonable situation, the modality-specific areas are involved in the forming of situation models, experiment two will be conducted. All of the sentences that are presented in the experiment two can form situation models, only the nouns are changed. For example, according to the description in experiment 
one "The teacher grabbed a pen on the desk", a similar sentence, which can form a situation model as well, will be presented in experiment two with only one word different from the previous one (e.g., The teacher grabbed a brush on the desk). We would like to make it clear that all of the texts presented in experiment 2 are reasonable. Besides, we use TMS to do the work.

\subsubsection{Materials and procedure:}

There are 24 sets of action descriptions. Stimulus materials are similar to those that are used in Experiment one. Half of them are hand-related actions and half of them are leg-related actions. The procedure is similar to experiment one, too. In experiment two, we use TMS instead of MRI, and the time recording is needless in experiment two. Transcranial magnetic stimulation should be applied to arm and leg loci over the left and right hemispheres during the comprehension of arm- and leg-related words.

\subsubsection{Prediction:}

- Modality-specific areas will be activated and the occipital cortex will play a role in forming situation models. We can conclude that the embodied cognition has a functional role in forming situation models under such prediction.

- In contrast, modality-specific areas will not be activated and the occipital cortex will not play a role in forming situation models, which cannot support that the embodied cognition plays a functional role in forming situation models

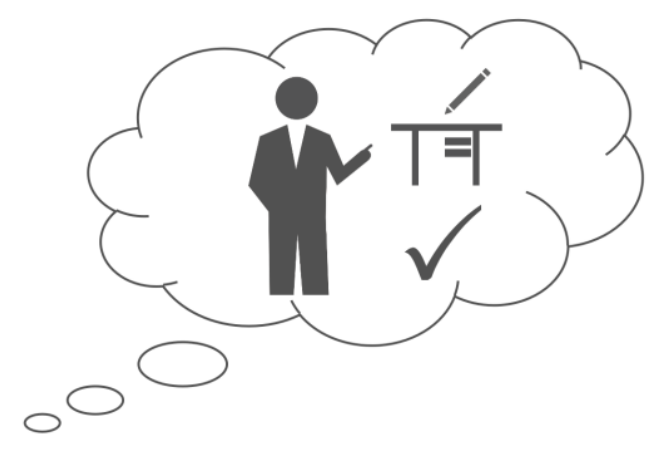

Figure 4 The reasonable situation model designed in experiment 2 , only with the material changed

\section{CONCERN ABOUT MEASURE INSTRUMENT}

In experiment one, we prepared MRI and time keeper. MRI performs digital image, which is much clearer for local capture. By comparing front and rear images, we are available to identify the extension of activity in modality-specific areas. Thus, we choose MRI to find out whether embodied cognition is functioning during the text-understanding. The fMRI is mainly used to observe changing images which means that it's hard to judge the formation of the situation model.

A time keeper is used to record the subject's reaction time. From discussions by other researchers, the reaction time required by readers for reasonable segments should be shorter. (e.g. Zwaan, 1999) Thus enabling us to detect the formation of situation model in this way.

For the experiment one, we are worried that MRI scan takes too long and may not synchronizes with the time keeper.

In experiment two, all the information that subjects received are reasonable, we only intended to make sure that the active areas changed as diverse texts presented. We decided to use TMS so that any change of the concerned areas can be found instantly.

In general, we use MRI for Experiment one and TMS for Experiment two. PET cannot work properly because it is not as accurate as MRI. However, the role of fMRI remains unclear and because we do not need to focus on the change of the brain, we finally decide to use TMS instead of fMRI.

\section{CONCLUSION}

We believed that the verified-results of our experiments will help people strengthen their learning, understanding and memory skills. Once the causal relationship between situation model and embodied cognition is confirmed, people will be more aware of how important the inputting memory of concrete stuff is, for those who have more situation-related memory will find it easier to form situation model. For example, when students find something difficult to understand and simulate, they can refer to the tangible things in reality to help them form imagery. In addition, find the forming mechanism of situation model can also help cure some diseases that are associated with difficulties to form situation model, which disturb people to strengthen their understanding to a text.

\section{ACKNOWLEDGMENT}

This paper was supported by Daniel Casasanto and Minhui Ni.

\section{REFERENCES}

[1] Carreiras, M., Garnham, A., Oakhill, J., \& Cain, K. (1996). The use of stereotypical gender information in constructing a mental model: Evidence from English and Spanish. Quarterly Journal of Experimental Psychology, 49A, 639-663.

[2] Daniel G. Morrow \& Herbert H. Clark (1988) Interpreting words in spatial descriptions, Language and Cognitive Processes, 3:4, 275-291. 
[3] Zwaan, R. A., (1999). Situation models: the metal leap into imagined worlds. Current Directions in Psychological Science, 8.

[4] Glenberg, A.M., Meyer, M., \& Lindem, K. (1987). Mental models contribute to foregrounding during text comprehension. Journal of Memory and Language, 26, 69-83.

[5] Morrow, D.G., Greenspan, S.L., \& Bower, G.H. (1987). Accessibility and situation models in narrative comprehension. Journal of Memory and Language, 26, 165-187.

[6] Simmons, W. K., Hamann, S. B., Harenski, C. L., Hu, X. P., \& Barsalou, L. W.. (2008). Fmri evidence for word association and situated simulation in conceptual processing. Journal of Physiology - Paris, 102(1-3), 106-119.

[7] Morrow, D.G., \& Clark, H.H. (1988). Interpreting words in spatial descriptions. Language and Cognitive Processes, 3, 275-291.

[8] Morrow, D. G., Bower, G. H., \& Greenspan, S. L., (1989). Updating situation models during narrative comprehension. Journal of Memory \& Language, 28(3), 292-312.

[9] Trabasso, T., \& Suh, S. (1993). Understanding text: Achieving explanatory coherence through online inferences and mental operations in working memory. Discourse Processes, 16, 3-34.

[10] Zwaan, R.A. (1996). Processing narrative time shifts. Journal of Experimental Psychology: Learning, Memory, and Cognition, 22, 1196-1207.

[11] Lawrence W. Barsalou, W. Kyle Simmons, Aron K. Barbey, Christine D. Wilson (2003), Grounding conceptual knowledge in modality-specific systems, Trends in Cognitive Sciences, 7, 84-91.

[12] Pulvermüller, F. (2005). Brain mechanisms linking language and action. Nat Rev Neurosci 6, 576582.J. Clerk Maxwell, A Treatise on Electricity and Magnetism, 3rd ed., vol. 2. Oxford: Clarendon, 1892, pp.68-73.

[13] Barsalou, L. W., Simmons, W. K. , Barbey, A. K. , \& CD Wilson. (2003). Grounding conceptual knowledge in modality-specific systems. Trends in Cognitive Sciences, 7(2), 84-91.

[14] Loftus, E. F., \& Palmer, J. C. (1974). Reconstruction of auto-mobile destruction: An example of the interaction between language and memory. Journal of Verbal Learning and Verbal behavior, 13, 585-589.

[15] Cosmides, L., \& Tooby, J.(1994). Better than Rational: Evolutionary Psychology and the
Invisible Hand.The American Economic Review,84[2),327-332. Retrieved December 26, 2020, from http://wwwjstororg/stable/2117853

[16] Dijk, T., \& Kintsch, W. (1983, January 1). Strategies of discourse comprehension. undefined. https://www.semanticscholar.org/paper/Strategiesof-discourse-comprehension-DijkKintsch/0d24df6d9507feeacdce5ff0633f95127fc1a 77c. 